\title{
The first galaxies: instrument requirements and concept study for OWL
}

\author{
J.-G. Cuby ${ }^{1}$, J.-P. Kneib ${ }^{1}$, F. Hammer ${ }^{2}$, E. Prieto ${ }^{1}$, M. Marteaud ${ }^{2}$, \\ P. Vola ${ }^{2}$, P. Jagourel ${ }^{2}$, P.-E. Blanc ${ }^{1}$ and T. Fusco ${ }^{3}$ \\ ${ }^{1}$ LAM, Laboratoire d'Astrophysique de Marseille, 13376 Marseille Cedex 12, France \\ email:jean-gabriel.cuby@oamp.fr \\ ${ }^{2}$ GEPI, Observatoire Paris-Meudon, 92195 Meudon, France \\ ${ }^{3}$ ONERA, BP72, 92322 Chatillon Cedex, France
}

\begin{abstract}
A highlight science case for the European ELT is: First light - The First Galaxies and the Ionization State of the Early Universe. It aims at understanding the formation and evolution of the first sources of light at the end of the Dark Ages and of the re-ionization of the Universe. The corresponding instrument requirements are: a few tens of integral field units with spatial sampling $\sim 20$ mas and individual fields of $\sim 1^{\prime \prime}$ over a wide field of view of $5^{\prime} \times 5^{\prime}$ or larger. MultiObject Adaptive Optics is required to locally provide significant image quality enhancement. Spectroscopic observations are required in the near IR domain with a spectral resolution of a few 1000. MOMFIS is a preliminary instrument concept designed for OWL around this science case. The instrument concept and preliminary design are presented. Development efforts are estimated, as well as development risks and required $\mathrm{R} \& \mathrm{D}$ activities.
\end{abstract}

Keywords. early universe, instrumentation: adaptive optics, spectrographs

\section{Introduction}

As part of the OWL conceptual design study ESO launched 8 instrument studies in 2005. One of these studies was for a Multi-Object, Multi-Field Infrared Spectrograph: MOMFIS. The scope of these OWL instrument studies is described in Monnet et al. (2006). The scientific requirements were derived from a detailed analysis of the science case. A conceptual opto-mechanical design was performed which allowed to assess feasibility, cost, risk and performance issues while providing feedback to the OWL designers.

What is the nature of the first objects in the Universe, how did they form and grow, how did they ionize and enrich the neutral gas surrounding them? ELTs will allow the observation of statistical samples of these first light objects with unprecedented depth and details. The requirement specifications for this particular science case are described in the following sections, targeting high redshift objects in the range $7<z<-15$ approximately. See also Bergeron (2006) for a thorough analysis of this and other cosmological science cases.

\section{Requirement specification}

\subsection{Multiplex, FoV \& sensitivity}

Extrapolation of the properties of galaxies at $z \sim 6$ as observed with $8-10 \mathrm{~m}$ class telescopes combined with models of galaxy formation allows to estimate the number counts of high$z$ galaxies versus $\mathrm{AB}$ magnitude in the redshift range 7 to 15 (see Figure 1). From these figures one derives that 20 to 50 sources at the very least, possibly a few hundreds, can 

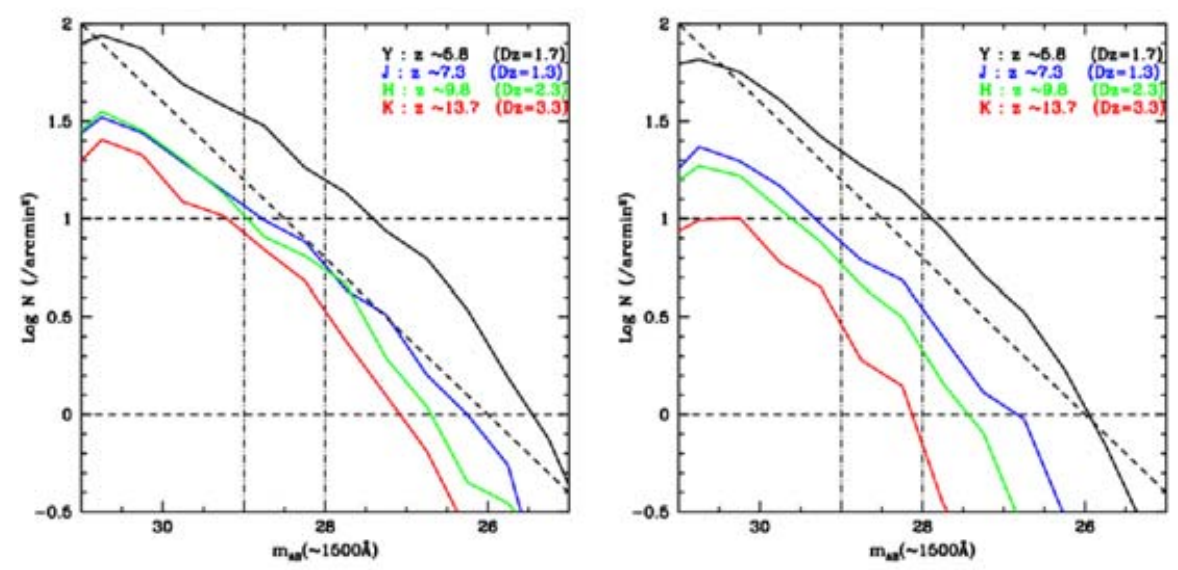

Figure 1. High- $z$ galaxy luminosity function. Counts prediction from extrapolation of the observed $z=6$ luminosity function (Bouwens et al. 2004a, Stiavelli et al. 2004).

Left: no evolution, Right: with moderate evolution. The number counts refer to the $1500 \AA$ rest frame UV luminosity. The redshift interval per spectral band is indicated.
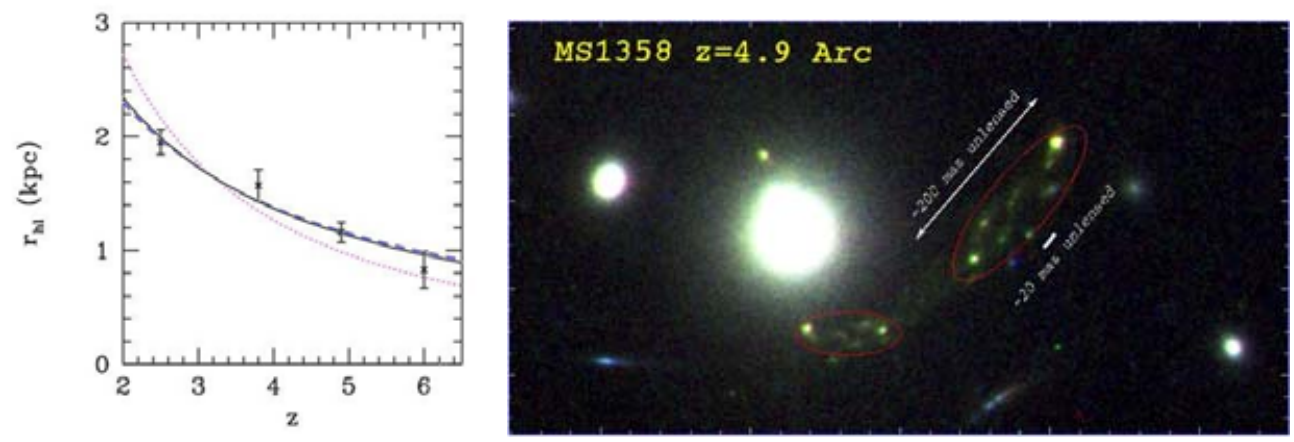

Figure 2. Left: Mean half light radius versus redshift. Measured from HST data on dropout samples of galaxies of fixed luminosity $\left(0.3-1.0 \mathrm{~L}^{*}, z=3\right)$ (Bouwens et al. 2004b). Right: HST/ACS color image (RIZ) of the MS1358+62 arc at $z=4.9$. The arc is made of 2 images as indicated by the 2 red ellipses. Due to the large magnification of $\sim 20$ (for the largest ellipse) the unlensed size of the images is about 200mas. Note that the arc displays a very complex structure of 7-8 blobs, each of them having a typical size of about 20mas (Kneib et al. 2006, in preparation).

be observed in a single $5^{\prime} \times 5^{\prime}$ field of view, depending on the observed band, effects of evolution, cosmic variance, etc., down to a sensitivity limit of $\mathrm{AB}=28$.

\subsection{Image quality, spatial sampling and individual Fo $V$}

Figure 2 (left) shows the typical size of galaxies versus redshift. Simple extrapolation of this curve at higher redshifts indicates that the half-light radius as $z=6$ is $\sim 1 \mathrm{kpc}$ and $0.5 \mathrm{kpc}$ at $z=8$, corresponding to angular sizes on sky of 170 and 100mas, respectively. Therefore, sampling the half light diameters with $\sim 10 \times 10$ spatial elements requires a spatial sampling of about 20 mas, requiring in turn an exquisite image quality (AO corrected) within 50mas or less. Figure 2 (right) nicely illustrates the need for integral field spectroscopy on a real image of a strongly magnified high-redshift object. 


\subsection{Spectral coverage and spectral resolution}

Because Ly $\alpha$ lies above $1 \mu \mathrm{m}$ for redshifts above $\sim 7$ and because thermal background affects on-ground performance above $\sim 2.4 \mu \mathrm{m}$, the spectral domain relevant to this science case is [1.0-2.4] $\mu \mathrm{m}$ approximately. For digital $\mathrm{OH}$ suppression the spectral resolution shall be $\sim 4000$ or higher - not considering here the potential for $\mathrm{OH}$ suppression devices. This spectral resolution is high enough to resolve velocity fields of the order of a few hundred $\mathrm{km} / \mathrm{s}$ typical of high-redshift galaxies.

\section{Discussion}

The requirements on number of targets calls for multi-object spectroscopy. The requirement on individual field of view and spatial sampling calls for integral field spectroscopy. The ideal instrument is therefore a multi-integral field spectrograph.

The requirement on spatial sampling (50mas) calls for adaptive optics correction. It is not possible to achieve adaptive optics correction over a field of view of several arcminutes in diameter, however the correction only needs to be local along the line of sight of the observed targets. This so-called Multi-Object Adaptive Optics (MOAO) is further described in Hubin \& Ellerbroek (2006). If reaching the diffraction limit of an ELT is not required, reaching the required 50mas image quality still calls for high density deformable mirrors and several laser guide stars to provide bright enough reference stars (for good sky coverage, which is an essential requirement for multi-wavelength cosmological fields observed by other facilities).

MOAO has not yet been developed or used at any telescope in the world, and it needs to be demonstrated. Backup/alternative/intermediate solutions to having a full blown MOAO system operating with laser guide stars are numerous: 1) no MOAO, but Ground Layer Adaptive Optics (GLAO) correction over a wide field, 2) no MOAO, but Multi-Conjugate Adaptive Optics (MCAO) over a smaller field, 3) low-order MOAO with natural guide stars. Further trade-off analyses including scientific performance and adaptive optics development plans are required to derive an implementation road map for any ELT MOAO system.

\section{Instrument concept and performance}

The MOMFIS instrument features 30 fully identical and independent channels with 10 cryostats, 3 spectrographs per cryostat. The instrument is modular, highly redundant, and designed for easy preventive or corrective maintenance. The principle of operation, overall design and implementation in the OWL focal station is shown Figure 3. The instrument consists of (per channel):

- A target selection system consisting of pick-off and beam steering mirrors which directs the science beams from the telescope focal plane to the deformable mirrors. Pickoff mirrors are put in place (position and tilt angle) in the focal plane by a positioner.

- A deformable mirror for AO correction in the direction of the target.

- An image slicer dividing. individual fields of view into 40 slices 20 mas wide

- A spectrograph providing one spectral band $(Y$ to $K)$ at once at a spectral resolution of $>4000$.

- A $2 \mathrm{k} \times 2 \mathrm{k}$ IR array. Options for $1 \mathrm{k} \times 1 \mathrm{k}$ detectors can be considered to reduce cost.

In addition, the instrument is equipped with wavefront sensors, which sample the atmosphere over the whole instrument field of view.

Figure 4 illustrates the performance capabilities of OWL. Reaching $J_{A B}=28$ on the continuum in a few hours of time is feasible provided that the PSF is very sharp. 

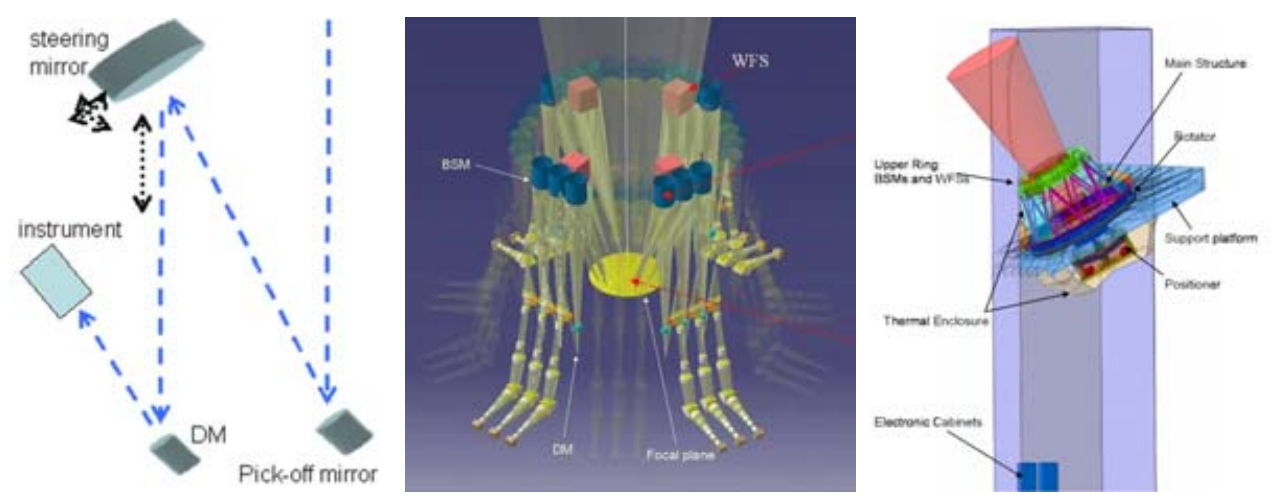

Figure 3. Left: operational concept. Pickoff mirrors adequately positioned and oriented in the telescope focal plane send the optical beams to adjustable beam steering mirrors which in turn send the beams to the fixed instruments (deformable mirrors, atmospheric dispersion compensators, image slicers, spectrographs). Middle: optical layout showing the optical path, from pickoff mirrors (focal plane) to beam steering mirrors (cylinders), DM, ADC, slicer unit and spectrograph. Right: overall implementation of the complete instrument in the OWL focal station. The overall height of the instrument as shown is $\sim 5 \mathrm{~m}$, the height of the focal station is $12 \mathrm{~m}$.

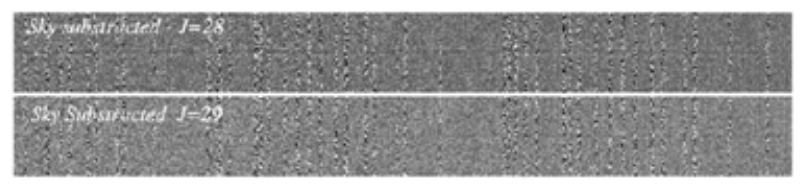

Figure 4. Simulated spectra of objects with $J=28$ and $J=29$. Spectral resolution is 4000, integration time $10 \mathrm{ksec}$, PSF $150 \mathrm{mas}$, telescope diameter $100 \mathrm{~m}$. This simulation, while illustrating that reaching $J=28$ is feasible, also shows the importance of image quality.

2D spectroscopy of objects extended or structured within $\sim 150$ mas will typically take up to 10-30 hours of integration time with a $100 \mathrm{~m}$ telescope, and up to a few hundreds of hours with smaller telescopes (30 to $50 \mathrm{~m}$ ). Targets will ideally come from JWST or from OWL diffraction limited imaging in the MCAO field of view.

\section{Conclusions}

MOMFIS allows the simultaneous observation of 30 targets in integral field mode in the $5^{\prime}$ OWL field of view down to IR AB magnitudes of $\sim 28$. This ideally meets the science high level specifications. The entire instrument concept relies on existing and well demonstrated technologies, but for 2 items which will require specific developments and roadmaps: 1) multi-object adaptive optics (MOAO). MOAO is at the core of the MOMFIS operation, it requires several wavefront sensors sampling the atmospheric wavefront over the telescope and one deformable mirror per channel (assuming the telescope provides ground layer AO correction). The MOAO concept has never been implemented and needs further studies and laboratory and/or on-sky prototyping to be demonstrated and validated. Laser guide stars are a must for full sky coverage. In a first phase, the instrument could operate without MOAO and be upgraded later. 2) Internal metrology. Internal metrology is required in the instrument to monitor and control precisely the optical paths. Internal metrology is also required for alignment, calibration and possibly control of the optical elements. 


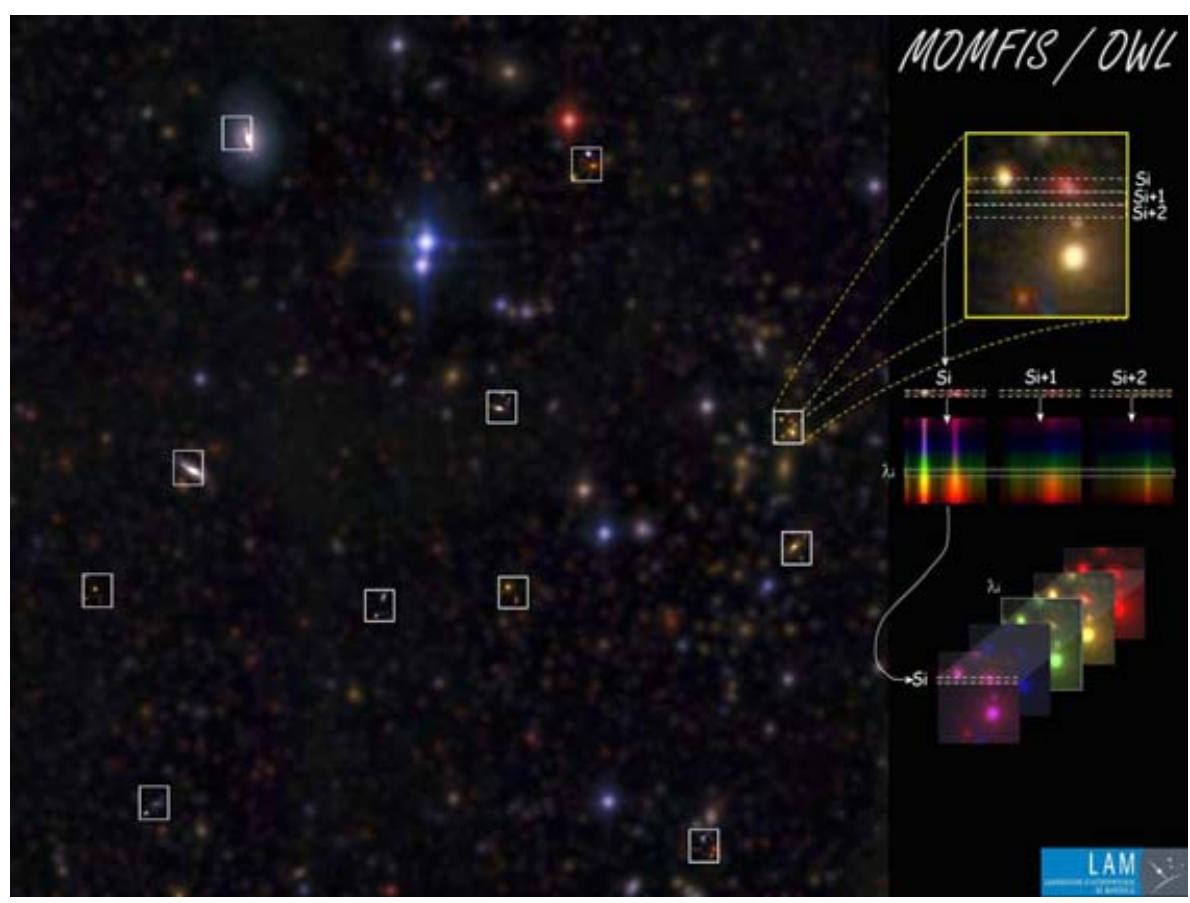

Figure 5. Illustration of the MOAO+IFU spectroscopy. Adaptive Optics correction is performed in the direction of the targets and each target beam is directed to an Integral Field Spectrograph

Several design and implementation options can be contemplated: progressive MOAO implementation, partial cryogenic cooling, smaller individual fields of view, etc. Alternative designs have been considered. They could take the form of traditional multi-slit spectrographs (MOS) or (OH suppression) fiber-fed spectrographs still requiring the pickoff and adaptive optics stages. MOMFIS is a complex instrument. Its development and integration will require a broad range of expertise and facilities across Europe. The hardware cost is estimated to be in the range 20-40 MEuros, depending on the selected options, and the required manpower (at institutes) in the range 150-250 person-years. The instrument development requires 10 years, including a few years of continuing R\&D activities. Figure 5 illustrates the MOMFIS principle of operation.

\section{References}

Monnet, G. et al. 2006, These Proceedings

Bergeron, J. et al. 2006, These Proceedings

Hubin, N. \& Ellerbroek, B. 2006, These Proceedings

Bouwens, R.J., Illingworth, G.D., Thompson, et al. 2004a, ApJ 606, L25

Bouwens, R.J., Illingworth, G.D., Blakeslee, J.P., Broadhurst, T.J. \& Franx, M. 2004b, ApJ 611, L1

Stiavelli, M., Fall, S.M. \& Panagia, N. 2004, ApJ 600, 508 